\title{
A TECHNIQUE FOR COMPUTING THE PDFS AND CDFS OF NONNEGATIVE INFINITELY DIVISIBLE RANDOM VARIABLES
}

\author{
MARK S. VEILLETTE*** AND \\ MURAD S. TAQQU, ${ }^{* * * *}$ Boston University
}

\begin{abstract}
We present a method for computing the probability density function (PDF) and the cumulative distribution function (CDF) of a nonnegative infinitely divisible random variable $X$. Our method uses the Lévy-Khintchine representation of the Laplace transform $\mathrm{Ee}^{-\lambda X}=\mathrm{e}^{-\phi(\lambda)}$, where $\phi$ is the Laplace exponent. We apply the PostWidder method for Laplace transform inversion combined with a sequence convergence accelerator to obtain accurate results. We demonstrate this technique on several examples, including the stable distribution, mixtures thereof, and integrals with respect to nonnegative Lévy processes.
\end{abstract}

Keywords: Infinitely divisible distribution; Post-Widder formula; stable distribution; stochastic integration

2010 Mathematics Subject Classification: Primary 60E07; 65C50; 60-08

Secondary 60-04

\section{Introduction}

Let $X$ be a nonnegative random variable. The distribution of $X$ is said to be infinitely divisible if, for any positive integer $n$, we can find independent and identically distributed (i.i.d.) random variables $X_{i, n}, i=1,2, \ldots, n$, such that $X \stackrel{\mathrm{D}}{=} X_{1, n}+X_{2, n}+\cdots+X_{n, n}$. For background, see [3] and [22]. There are many examples of such distributions, including the gamma distribution, compound Poisson distributions, the inverse Gaussian distribution, and right-skewed stable distributions. These distributions are also central in the study of nondecreasing Lévy processes. In this paper we give a method for numerically computing the probability density function (PDF) and the cumulative distribution function $(\mathrm{CDF})$ of a nonnegative infinitely divisible random variable.

Our starting point is the Lévy-Khintchine (LK) formula (see [4], [5], and [21]), which in the case of nonnegative infinitely divisible random variables states that the Laplace transform of $X$,

$$
\psi(\lambda) \equiv \mathrm{Ee}^{-\lambda X}=\mathrm{e}^{-\phi(\lambda)}, \quad \lambda>0,
$$

has an exponent $\phi(\lambda)$ which is called the Laplace exponent, which can be written as

$$
\phi(\lambda)=a \lambda+\int_{0}^{\infty}\left(1-\mathrm{e}^{-\lambda u}\right) \Pi(\mathrm{d} u)
$$

Received 27 May 2010; revision received 3 November 2010.

This work was partially supported by the NSF grants DMS-0706786 and DMS-1007616 at Boston University.

* Postal address: Department of Mathematics, Boston University, 111 Cummington Street, Boston, MA 02215, USA.

** Email address: mveillet@bu.edu

*** Email address: murad@bu.edu 
Here, $a \geq 0$ is a shift and $\Pi$ is a measure on $(0, \infty)$ which satisfies

$$
\int_{0}^{\infty}(1 \wedge x) \Pi(\mathrm{d} x)<\infty .
$$

Since we are interested primarily in PDFs and CDFs, we will assume that $a=0$ throughout, since a positive $a$ only shifts the PDF/CDF. Let $f_{X}(x)$ and $F_{X}(x)$ denote the PDF and CDF of $X$, respectively (we will promptly drop the subscript $X$ on these functions when it is clear from the context). The Laplace transforms of the PDF and CDF can be easily obtained from the LK formula (we will use a tilde to denote a Laplace transform):

$$
\psi(\lambda)=\tilde{f}(\lambda)=\int_{0}^{\infty} \mathrm{e}^{-\lambda x} f(x) \mathrm{d} x,
$$

and a simple application of Fubini's theorem implies that

$$
\Psi(\lambda) \equiv \tilde{F}(\lambda)=\int_{0}^{\infty} \mathrm{e}^{-\lambda x}\left(\int_{0}^{x} f(y) \mathrm{d} y\right) \mathrm{d} x=\frac{1}{\lambda} \int_{0}^{\infty} \mathrm{e}^{-\lambda y} f(y) \mathrm{d} y=\frac{\psi(\lambda)}{\lambda} .
$$

Thus, obtaining $f$ and $F$ is a matter of inverting a Laplace transform. Generally, this task is not easy. Typically, it is done by complex integration of the Laplace transform (see [1]), which can be difficult if the integrands are slowly decaying, oscillatory functions. This causes many numerical integration methods to converge slowly. Here, we apply a different method of Laplace inversion known as the Post-Widder (PW) method (see [2, Theorem 2] or [7, Section VII.6]). It is based on the fact that, under weak conditions on a function $f$, we have

$$
f(x)=\lim _{k \rightarrow \infty} \frac{(-1)^{k-1}}{(k-1) !}\left(\frac{k}{x}\right)^{k} \tilde{f}^{(k-1)}\left(\frac{k}{x}\right), \quad x>0,
$$

where $\tilde{f}^{(k-1)}(k / x)$ denotes the $(k-1)$ th derivative of $\tilde{f}$ evaluated at $k / x$. Thus, instead of integrating the Laplace transform, we are taking arbitrarily high derivatives.

The obvious challenge in using this method is computing high derivatives of $\tilde{f}$ in (1.4) to approximate the limit. Some methods have been developed to do this in general (see, for instance, [1] or [10]); however, most involve complex integration. The method we describe here for the case of infinitely divisible distributions is both easy to implement and only involves (at worst) integration of a real-valued nonnegative exponentially decaying function. The method we use here combines both computation of a finite number of terms of the sequence in (1.4) and a numerical extrapolation method to approximate the limit; see [8]. A similar method was used to compute mean first passage times of Lévy subordinators in [24].

This paper is organized as follows. In Section 2 we give an overview of the approximation method used based on the PW formula. We test the method in cases where the PDF and CDF are known in closed form in Section 3. In Section 4 we apply our method to a collection of examples. Finally, in Appendix A we outline the algorithm for finding $f$ and $F$, as well as numerical issues that may arise in the computation.

\section{Post-Widder method with extrapolation}

Given a continuous function $g(x), x>0$, which is bounded as $x \rightarrow \infty$, we will denote its $k$ th PW approximation as

$$
g_{k}(x)=\frac{(-1)^{k-1}}{(k-1) !}\left(\frac{k}{x}\right)^{k} \tilde{g}^{(k-1)}\left(\frac{k}{x}\right), \quad x>0 .
$$




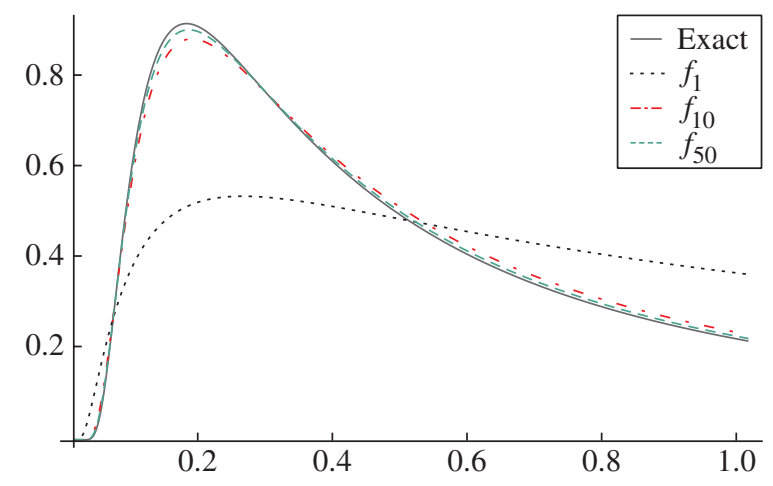

FIgURE 1: The exact form of $f$ together with the PW approximations $f_{1}, f_{10}$, and $f_{50}$.

The fact that $g_{k}(x) \rightarrow g(x)$ as $k \rightarrow \infty$ can be seen by approximating $g(x)$ by $\operatorname{E} g\left(\bar{Y}_{k}\right)$, where $\bar{Y}_{k}=k^{-1}\left(Y_{1}+Y_{2}+\cdots+Y_{k}\right)$, and the $Y_{i}$ s are i.i.d. gamma random variables with mean $x$ and variance $x^{2}$, and then applying the law of large numbers (see Section VII.6 of [7]).

The convergence of $g_{k}(x)$ to $g(x)$ is slow in general. To illustrate this point, let $g$ be a PDF $f$ of an inverse Gaussian distribution for which the Laplace transform is given by $\tilde{f}(\lambda)=\exp \left(-\lambda^{-1 / 2}\right.$ ) (in this case the density $f(x)$ is known in closed form-see Section 3 ). In Figure 1 we plot the exact formula for $f$ as well as $f_{k}$ for $k=1,10$, and 50. Note that even with 49 derivatives of $\tilde{f}$, the approximation is still poor. In fact, this is a general feature of the PW formula, as it has been shown (see [10]) that the errors $\varepsilon_{k}(f ; x) \equiv f(x)-f_{k}(x)$ have a power series expansion:

$$
\varepsilon_{k}(f ; x)=\sum_{m=1}^{\infty} \frac{a_{m}(x)}{k^{m}} .
$$

Here the coefficients $a_{m}(m)$ are given by

$$
a_{m}(x)=\sum_{j=1}^{m} f^{(j+m)}(x) x^{j+m} \frac{d(j+m, m)}{(j+m) !}
$$

and the $d(j+m, m)$ are the associated Stirling numbers of the first kind (see [18, Chapter 4, Section 4]). Thus, the convergence of $f_{k}$ is in general $O(1 / k)$ corresponding to the $m=1$ term. This method alone is thus inadequate for computing the inverse Laplace transform to a high level of precision.

To obtain high precision, it is necessary to couple the PW approximations with a convergence acceleration method which extrapolates the limit in (1.4) based on a finite collection of terms in the sequence. Thus, our method for computing the PDF $f$ at $x>0$ involves two components:

1. for some sequence $k_{1}<k_{2}<\cdots<k_{N}$, compute the approximates $f_{k_{i}}(x)$;

2. use the points $\left(k_{1}, f_{k_{1}}(x)\right), \ldots,\left(k_{N}, f_{k_{N}}(x)\right)$ to extrapolate $\lim _{k \rightarrow \infty} f_{k}(x)$.

For the CDF, replace $f$ with $F$ above. We focus first on step 1, which in view of (2.1) involves computing high derivatives of $\psi$ or $\Psi$. Next, we will discuss two methods of convergence acceleration to address step 2 . 
Remark. Our method produces the best results if the PDF and/or CDF are smooth functions for $x>0$. That being said, this method still produces useful results in the nonsmooth case; however, it may fail to converge near points where the function lacks smoothness. This method should not be used for distributions which contain atoms, such as the Poisson distribution, or even distributions whose density lacks smoothness, such as a compound Poisson distribution with bounded jump distribution. For such distributions, another method based on numerically solving a Kolmogorov-Feller forward equation, is more suitable; see [23].

\subsection{Computing derivatives}

2.1.1. Derivatives of $\psi$. We begin by presenting two methods for computing the derivatives of $\psi(\lambda)=\exp (-\phi(\lambda))$. The first method is based on the simple observation that

$$
\psi^{\prime}(\lambda)=-\phi^{\prime}(\lambda) \psi(\lambda)
$$

Thus, Leibnitz's formula implies that, for any $k \geq 1$, the $(k+1)$ th derivative of $\psi$ is given by

$$
\psi^{(k+1)}(\lambda)=-\sum_{j=0}^{k}\left(\begin{array}{l}
k \\
j
\end{array}\right) \psi^{(j)}(\lambda) \phi^{(k+1-j)}(\lambda)
$$

Note that, for fixed $\lambda$, the values of $\psi(\lambda), \psi^{\prime}(\lambda), \ldots, \psi^{(k)}$ can be computed recursively using (2.3) if one has first computed $\phi^{(j)}(\lambda)$ for $j=1,2, \ldots, k+1$.

Alternatively, the $k$ th derivative of $\psi(\lambda)=\exp (-\phi(\lambda))$ can be given directly in terms of the derivatives of $\phi$ using Faá di Bruno's formula (see [18, Chapter 2, Section 8]), which in our case yields

$$
\psi^{(k)}(\lambda)=\frac{\mathrm{d}^{k}}{\mathrm{~d} \lambda^{k}} \mathrm{e}^{-\phi(\lambda)}=\mathrm{e}^{-\phi(\lambda)} \sum \frac{k !}{m_{1} ! m_{2} ! \cdots m_{k} !}\left(\frac{-\phi^{(1)}(\lambda)}{1 !}\right)^{m_{1}} \cdots\left(\frac{-\phi^{(k)}(\lambda)}{k !}\right)^{m_{k}},
$$

where the sum runs over all $m_{1}, \ldots, m_{k}$ such that $m_{1}+2 m_{2}+\cdots+k m_{k}=k$. This can be expressed more simply in terms of the $k$ th complete Bell polynomial, $B_{k}\left(x_{1}, x_{2}, \ldots, x_{k}\right)$, as

$$
\psi^{(k)}(\lambda)=\mathrm{e}^{-\phi(\lambda)} B_{k}\left(-\phi^{(1)}(\lambda),-\phi^{(2)}(\lambda), \ldots,-\phi^{(k)}(\lambda)\right) .
$$

See [17] or [19]. Furthermore, $B_{k}$ can be given in terms of the following $k \times k$ determinant:

$$
B_{k}\left(x_{1}, \ldots, x_{k}\right)=\left|\begin{array}{cccccc}
x_{1} & \left(\begin{array}{c}
k-1 \\
1
\end{array}\right) x_{2} & \left(\begin{array}{c}
k-1 \\
2
\end{array}\right) x_{3} & \left(\begin{array}{c}
k-1 \\
3
\end{array}\right) x_{4} & \cdots & x_{k} \\
-1 & x_{1} & \left(\begin{array}{c}
k-2 \\
1
\end{array}\right) x_{2} & \left(\begin{array}{c}
k-2 \\
2
\end{array}\right) x_{3} & \ldots & x_{k-1} \\
0 & -1 & x_{1} & \left(\begin{array}{c}
k-3 \\
1
\end{array}\right) x_{2} & \cdots & x_{k-2} \\
0 & 0 & -1 & x_{1} & \cdots & x_{k-3} \\
\vdots & \vdots & \vdots & \ddots & \vdots \\
0 & 0 & 0 & \cdots & -1 & x_{1}
\end{array}\right| .
$$

This provides another way for computing $\psi^{(k)}$. 
Both methods above require the derivatives of the Laplace exponent $\phi$. Fortunately, these can be computed directly, since the LK formula implies that, for any $\lambda>0$,

$$
\phi^{(n)}(\lambda)= \begin{cases}\int_{0}^{\infty}\left(1-\mathrm{e}^{-\lambda u}\right) \Pi(\mathrm{d} u), & n=0, \\ (-1)^{n+1} \int_{0}^{\infty} u^{n} \mathrm{e}^{-\lambda u} \Pi(\mathrm{d} u), & n \geq 1 .\end{cases}
$$

Note that passing derivatives through the integrals above is justified by the integrability assumption (1.2), since, for any $n \geq 1, x^{n} \mathrm{e}^{-\lambda x} \leq C_{n, \lambda}(1 \wedge x)$ uniformly on $\{x>0\}$ for some constant $C_{n, \lambda}>0$. For some examples, these integrals have a closed-form expression and can be computed easily. If a closed form is not available, many numerical integration methods are effective as these integrands are nonnegative, exponentially decaying functions. In this case, it is important to use a small relative error tolerance, as $\phi^{(n)}(\lambda)$ can become extremely small for large $\lambda$.

2.1.2. Derivatives of $\Psi$. For $\Psi$ defined in (1.3), we again apply Leibnitz's formula to (1.3) and obtain

$$
\Psi^{(k)}(\lambda)=\tilde{F}^{(k)}(\lambda)=\frac{\mathrm{d}^{k}}{\mathrm{~d} \lambda^{k}} \frac{\psi(\lambda)}{\lambda}=\sum_{j=0}^{k}\left(\begin{array}{l}
k \\
j
\end{array}\right)(-1)^{j} \frac{j !}{\lambda^{j+1}} \psi^{(k-j)}(\lambda) .
$$

When combined with the Post-Widder formula (2.1), we see a good deal of cancellation:

$$
\begin{aligned}
F_{k}(x) & =\frac{(-1)^{k}}{(k-1) !}\left(\frac{k}{x}\right)^{k} \tilde{F}^{(k-1)}\left(\frac{k}{x}\right) \\
& =\frac{(-1)^{k-1}}{(k-1) !}\left(\frac{k}{x}\right)^{k} \Psi^{(k-1)}\left(\frac{k}{x}\right) \\
& =\frac{(-1)^{k-1}}{(k-1) !}\left(\frac{k}{x}\right)^{k}\left(\sum_{j=0}^{k-1}\left(\begin{array}{c}
k-1 \\
j
\end{array}\right)(-1)^{j} \frac{j !}{(k / x)^{j+1}} \psi^{(k-1-j)}\left(\frac{k}{x}\right)\right) \\
& =\sum_{j=0}^{k-1} \frac{(-1)^{k+j-1}}{(k-j-1) !}\left(\frac{k}{x}\right)^{k-j-1} \psi^{(k-j-1)}\left(\frac{k}{x}\right) .
\end{aligned}
$$

Note that this requires the values $\psi(\lambda), \psi^{\prime}(\lambda), \ldots, \psi^{(n)}(\lambda)$, which can be computed using (2.3) and (2.5); hence, the recursive formula (2.3) is more convenient here than (2.4) since it computes all derivatives of $\psi$.

2.1.3. Other useful derivatives. In some applications, the derivatives of the PDF are also of interest. Assuming that the first $q-1$ derivatives of $f$ vanish at 0 , i.e. $f(0)=f^{\prime}(0)=\cdots=$ $f^{(q-1)}(0)=0$, then $f^{(q)}(x)$ has Laplace transform

$$
\widetilde{f^{(q)}}(\lambda)=\lambda^{q} \psi(\lambda)-\lambda^{q-1} f(0)-\lambda^{q-2} f^{\prime}(0)-\cdots-f^{q-1}(0)=\lambda^{q} \psi(\lambda),
$$

where $\psi(\lambda)=\tilde{f}(\lambda)$. In which case, Leibnitz's formula gives

$$
\frac{\mathrm{d}^{k}}{\mathrm{~d} \lambda^{k}} \widetilde{f^{(q)}}(\lambda)=\frac{\mathrm{d}^{k}}{\mathrm{~d} \lambda^{k}} \lambda^{q} \psi(\lambda)=\sum_{j=0}^{q \wedge(k-1)}\left(\begin{array}{l}
k \\
j
\end{array}\right) \frac{q !}{(q-j) !} \lambda^{q-j} \psi^{(k-j)}(\lambda) .
$$

Thus, (2.3) and (2.5) can be applied to compute the derivatives of $f$. 


\subsection{Extrapolation}

We want to compute the PDF $f(x)$ or $\operatorname{CDF} F(x)$ by approximating the limit as $k \rightarrow \infty$ in the Post-Widder formula (1.4). By letting $h=k^{-1}$, (2.2) implies that $f(x)$ can be written as

$$
f(x)=f_{k}(x)+\sum_{m=1}^{\infty} a_{m} h^{m} .
$$

We shall truncate the error series $\sum_{m=1}^{\infty}$ at $m=N-1$ and consider nonconsecutive $k_{1}<k_{2}<$ $\cdots<k_{n}$. If $h_{i}=k_{i}^{-1}, i=1, \ldots, N$, we can use the points $\left(h_{1}, f_{k_{1}}(x)\right),\left(h_{2}, f_{k_{2}}(x)\right), \ldots$, $\left(h_{N}, f_{k_{N}}(x)\right)$ to estimate the first $N-1$ unknown coefficients $a_{m}$. Indeed, the (approximate) $N \times N$ system

$$
f_{k_{i}}(x) \approx f(x)-a_{1} h_{j}-a_{2} h_{j}^{2}-\cdots-a_{n} h_{j}^{N-1}, \quad j=1,2, \ldots, N,
$$

can be solved for the $N$ unknowns $f(x), a_{1}, \ldots, a_{N-1}$. Since we are only interested in $f(x)$, it is enough to compute only the first row of the inverse of the matrix corresponding to system (2.8). This is essentially done in the polynomial extrapolation method described below in order to obtain an accurate approximation of the $\operatorname{limit}_{k \rightarrow \infty} f_{k}(x)$. There is a vast literature on such extrapolation methods and the errors associated to them; see [11] for a review.

Below, we briefly review two techniques for approximating $f(x)$ also used in [8]. We call these methods of extrapolation. The first is based on polynomial interpolation and the second is based on rational function (Padé) interpolation. Not surprisingly, the rational extrapolation provides faster convergence in many cases; however, we found it more susceptible to numerical instability for larger $N$ and $k_{N}$. Therefore, the 'better' choice depends on the particular example and the desired accuracy.

2.2.1. Polynomial extrapolation. Given the points $\left(h_{1}, f_{k_{1}}(x)\right),\left(h_{2}, f_{k_{2}}(x)\right), \ldots,\left(h_{N}, f_{k_{N}}(x)\right)$, the $N-1$ degree Lagrange polynomial $P_{N}(x)$ which passes through these points is given by

$$
P_{N}(h)=\sum_{i=1}^{N}\left(\prod_{j \neq i} \frac{h-h_{j}}{h_{i}-h_{j}}\right) f_{k_{i}}(x) .
$$

Thus, taking $h=0$ (or $k=\infty$ ) in this polynomial provides the approximation

$$
f(x) \approx P_{N}(0)=\sum_{i=1}^{N} c_{i} f_{k_{i}}(x), \quad \text { where } \quad c_{i}=\prod_{j \neq i} \frac{-h_{j}}{h_{i}-h_{j}}=\frac{(-1)^{N-1} k_{i}^{N-1}}{\prod_{j \neq i}\left(k_{j}-k_{i}\right)} .
$$

We thus use a linear combination of the approximations $f_{k_{i}}(x)$ to obtain a more accurate approximation of $f(x)$.

2.2.2. Rational extrapolation. As an alternative to polynomial extrapolation, we may instead fit a rational function to the points $\left(h_{1}, f_{k_{1}}(x)\right), \ldots,\left(h_{N}, f_{N} k_{N}(x)\right)$ of the form $R_{N}(h)=$ $P_{\mu}(h) / Q_{\nu}(h)$, where $P_{\mu}(h)=\sum_{i=1}^{\mu} p_{i} h^{i}$ is a polynomial of degree $\mu=\lfloor N / 2\rfloor$ and $Q_{\nu}(h)=$ $\sum_{i=0}^{v} q_{i} h^{i}$ is a polynomial of degree $v=N-\lfloor N / 2\rfloor$. Here, the coefficients $p_{i}$ and $q_{i}$ are chosen so that $R_{N}\left(h_{i}\right)=f_{k_{i}}(x)$. When implementing this method, these coefficients are not computed directly like in the polynomial extrapolation case, but instead $R_{N}(0)$ is computed iteratively in a triangular array using the recursive formulae (see [11, Section 13])

$$
R_{-1}^{i}=0, \quad R_{0}^{i}=f_{k_{i}}\left(h_{i}\right), \quad i \geq 1, \quad R_{m}^{i}=R_{m-1}^{i+1}+\frac{R_{m-1}^{i+1}-R_{m-1}^{i}}{h_{i} \Theta_{m}^{i}-h_{i+m}},
$$

where $\Theta_{m}^{i}=\left(R_{m-1}^{i}-R_{m-1}^{i+1}\right) /\left(R_{m-1}^{i+1}-R_{m-2}^{i+1}\right)$. We then take $f(x) \approx R_{N}^{1}=R_{N}(0)$ as our 
approximation since $h=1 / k \rightarrow 0$ corresponds to $k \rightarrow \infty$. Note that, unlike the polynomial extrapolation, this transformation is nonlinear in the $f_{k_{i}}(x)$.

2.2.3. Error bounds. One major drawback to using extrapolation techniques is that the error made in the approximation is difficult to bound analytically (see, for instance, Theorem 3 of [6] and Equation (13) of [8]). We can, however, obtain an asymptotic error bound for a rational or polynomial approximation. Let $h_{1}<h_{2}<h_{3}<\cdots$ be a sequence for which the approximant $f_{k_{i}}(x)$ can be computed with $k_{i}=1 / h_{i}$. Also, let $P_{N}(0)$ be the approximation of $f(x)$ obtained by using polynomial extrapolation with $h_{1}, h_{2}, \ldots, h_{N}$ for $N \geq 2$ (all the following formulae also hold with rational extrapolation by replacing $P_{N}(0)$ with $R_{N}(0)$ ). In [6], Bulirsch and Stoer constructed a second estimate of $f(x), \tilde{P}_{N}(0)$, with the property that

$$
\lim _{N \rightarrow \infty} \frac{P_{N}(0)-f(x)}{\tilde{P}_{N}(0)-f(x)}=-1 .
$$

Thus, asymptotically, $\tilde{P}_{N}(0)$ is as good an approximation to $f(x)$ as $P_{N}(0)$, except that it approaches $f(x)$ from the opposite direction. This second approximation is obtained using a linear combination of the form

$$
\tilde{P}_{N}(0)=(1+\alpha) P_{N+1}(0)-\alpha P_{N}(0),
$$

and choosing the constant $\alpha$ to change the sign of the leading term in the error $P_{N}(0)-f(x)$. Bulirsch and Stoer showed that this constant $\alpha$ is given by

$$
\alpha=1+\frac{2}{h_{1} / h_{N+1}-1} .
$$

This allows us to construct a numerical bound on the relative error made in this method. From (2.9) we have

$$
\frac{P_{N}(0)-\tilde{P}_{N}(0)}{f(x)-\tilde{P}_{N}(0)}=\frac{P_{N}(0)-f(x)}{f(x)-\tilde{P}_{N}(0)}+1 \rightarrow 2 .
$$

Fix any $\eta \in\left(\frac{1}{2}, 1\right)$. From (2.12) and since $\eta>\frac{1}{2}$, there exists an $N_{0}(\eta)$ large enough such that, for $N>N_{0}(\eta),\left|f(x)-\tilde{P}_{N}(0)\right|<\eta\left|P_{N}(0)-\tilde{P}_{N}(0)\right|$. Using a similar argument, there also exists an $M_{0}(\eta)$ large enough such that, for $N>M_{0}(\eta),\left|P_{N}(0)-f(x)\right|<\eta\left|P_{N}(0)-\tilde{P}_{N}(0)\right|$. Moreover, since $P_{N}(0) \rightarrow f(x)$ and $\eta<1$, we can pick $N_{1}(\eta)$ large enough such that $\left|P_{N}(0) / f(x)\right|<1 / \eta$ for all $N>N_{1}(\eta)$.

We can thus refine our estimate as $f(x) \approx\left(P_{N}(0)+\tilde{P}_{N}(0)\right) / 2$. If $N>\max \left(N_{0}(\eta), M_{0}(\eta)\right)$,

$$
\begin{aligned}
\left|\frac{P_{N}(0)+\tilde{P}_{N}(0)}{2}-f(x)\right| & \leq \frac{\left|P_{N}(0)-f(x)\right|}{2}+\frac{\left|f(x)-\tilde{P}_{N}(0)\right|}{2} \\
& \leq \eta\left|P_{N}(0)-\tilde{P}_{N}(0)\right| \\
& \leq\left|P_{N}(0)-\tilde{P}_{N}(0)\right|,
\end{aligned}
$$

and, if $N>\max \left(N_{0}(\eta), M_{0}(\eta), N_{1}(\eta)\right)$,

$$
\begin{aligned}
\frac{\left|\left(P_{N}(0)+\tilde{P}_{N}(0)\right) / 2-f(x)\right|}{f(x)} & \leq \frac{\left|P_{N}(0)-f(x)\right|}{2 f(x)}+\frac{\left|f(x)-\tilde{P}_{N}(0)\right|}{2 f(x)} \\
& \leq \eta \frac{P_{N}(0)}{f(x)} \frac{\left|P_{N}(0)-\tilde{P}_{N}(0)\right|}{P_{N}(0)} \\
& \leq \frac{\left|P_{N}(0)-\tilde{P}_{N}(0)\right|}{P_{N}(0)} .
\end{aligned}
$$


We thus have the following result.

Theorem 2.1. Suppose that $f(x) \neq 0$, let $P_{N}(0)$ be an approximation of $f(x)$ obtained using either polynomial extrapolation or rational extrapolation, and let $\tilde{P}_{N}(0)$ be defined as in $(2.10)$. For large enough $N$, we have

$$
\left|\frac{P_{N}(0)+\tilde{P}_{N}(0)}{2}-f(x)\right| \leq\left|P_{N}(0)-\tilde{P}_{N}(0)\right|
$$

and

$$
\frac{\left|\left(P_{N}(0)+\tilde{P}_{N}(0)\right) / 2-f(x)\right|}{f(x)} \leq \frac{\left|P_{N}(0)-\tilde{P}_{N}(0)\right|}{P_{N}(0)},
$$

where the right-hand sides converge to 0 as $N \rightarrow \infty$.

This provides a numerical bound on the absolute and relative errors of the approximation and suggests increasing $N$ until either $\left|P_{N}(0)-\tilde{P}_{N}(0)\right|$ or $\left|P_{N}(0)-\tilde{P}_{N}(0)\right| / P_{N}(0)$ is smaller than a prescribed value.

\section{Testing the method}

In this section we use MATHEMATICA ${ }^{\circledR}$ and consider two examples for which it is possible to compute the PDF and CDF exactly. The first example is the chi-squared distribution $\chi^{2}$ with one degree of freedom. For this distribution,

$$
\begin{aligned}
f_{\chi^{2}}(x) & =\frac{1}{\sqrt{2 \pi}} x^{-1 / 2} \mathrm{e}^{-x / 2}, \\
F_{\chi^{2}}(x) & =\operatorname{erf}\left(\sqrt{\frac{x}{2}}\right) \\
\psi_{\chi^{2}}(\lambda) & =\tilde{f}_{\chi^{2}}(\lambda)=(1+2 \lambda)^{-1 / 2}, \\
\phi_{\chi^{2}}(\lambda) & =-\log \psi_{\chi^{2}}(\lambda)=\frac{1}{2} \log (1+2 \lambda)=\int_{0}^{\infty}\left(1-\mathrm{e}^{-\lambda u}\right) \frac{\mathrm{e}^{-u / 2}}{2 u} \mathrm{~d} u, \\
\phi_{\chi^{2}}^{(n)}(\lambda) & =(-1)^{n+1} \int_{0}^{\infty} u^{n} \mathrm{e}^{-\lambda n} \frac{\mathrm{e}^{-u / 2}}{2 u} \mathrm{~d} u \\
& =\frac{(-1)^{n+1}}{2} \int_{0}^{\infty} u^{n-1} \mathrm{e}^{-u(\lambda+1 / 2)} \mathrm{d} u \\
& =\frac{(-1)^{n+1}(n-1) !}{2}\left(\frac{1}{2}+\lambda\right)^{-n}, \quad n \geq 1 .
\end{aligned}
$$

Results over a range of inputs are shown in Table 1 . We show the $x$-values considered as well as the value of $N$ required to obtain a relative precision of $10^{-6}$ and $10^{-15}$. In Figure 2, we also plot the relative error as a function of $N$. Note that the value of $N$ required is substantially higher when the value of the PDF or CDF takes on very small values, in particular, when $f_{\chi^{2}}(50) \approx 7.83 \times 10^{-13}$. This is because $f_{k}(x)$ over approximates $f$ at these points. 
TABLE 1: PDFs and CDFs of the $\chi^{2}$ distribution. Using $k_{i}=10 i$, we give the value of $N$ required to obtain six digits of accuracy $\left(N_{6}\right)$ and 15 digits of accuracy $\left(N_{15}\right)$ at the given values of $x$ using polynomial interpolation. Note that it becomes harder to approximate these functions to a small relative error when they become very small.

\begin{tabular}{ccrrccc}
\hline \multirow{2}{*}{$x$} & \multicolumn{3}{c}{ PDF } & \multicolumn{3}{c}{ CDF } \\
\cline { 2 - 7 } & $f_{\chi^{2}}(x)$ & $N_{6}$ & $N_{15}$ & $F_{\chi^{2}}(x)$ & $N_{6}$ & $N_{15}$ \\
\hline $10^{-5}$ & $1.26 \times 10^{2}$ & 4 & 8 & $2.52 \times 10^{-3}$ & 3 & 8 \\
$10^{-1}$ & 1.20 & 4 & 8 & 0.248 & 3 & 8 \\
1 & 0.242 & 4 & 9 & 0.683 & 3 & 8 \\
10 & $8.50 \times 10^{-4}$ & 6 & 12 & 0.998 & 4 & 11 \\
20 & $4.05 \times 10^{-6}$ & 9 & 15 & $1-7.7 \times 10^{-6}$ & 4 & 11 \\
50 & $7.83 \times 10^{-13}$ & 14 & 21 & $1-1.54 \times 10^{-13}$ & 1 & 12 \\
\hline
\end{tabular}

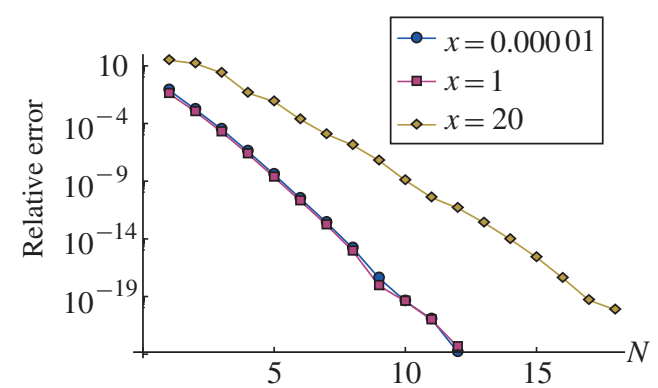

(a)

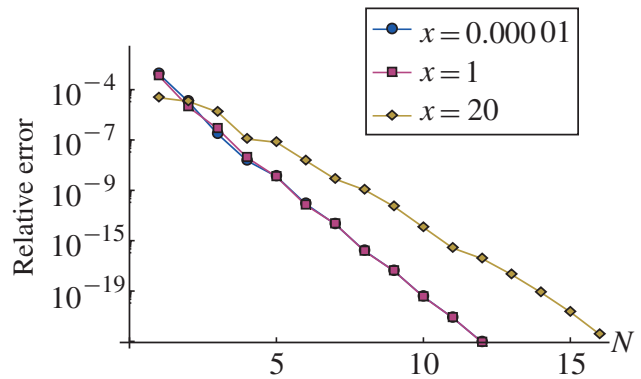

(b)

Figure 2: Plot of the relative error of our method versus the truncation level $N$ for the (a) PDF and (b) $\mathrm{CDF}$ of the $\chi^{2}$ distribution at three values of $x$.

The second example we considered is the inverse Gaussian (IG) distribution (see [3, Example 1.3.21]), for which

$$
\begin{aligned}
f_{\mathrm{IG}}(x) & =\frac{1}{\sqrt{4 \pi x^{3}}} \mathrm{e}^{-1 /(4 x)}, \\
F_{\mathrm{IG}}(x) & =1-\operatorname{erf}\left(\frac{1}{\sqrt{2 x}}\right), \\
\psi_{\mathrm{IG}}(\lambda) & =\tilde{f}_{\mathrm{IG}}(\lambda)=\mathrm{e}^{-\sqrt{\lambda}}, \\
\phi_{\mathrm{IG}}(\lambda) & =-\log \psi_{\mathrm{IG}}(\lambda)=\sqrt{\lambda}=\int_{0}^{\infty}\left(1-\mathrm{e}^{-\lambda u}\right) \frac{u^{-3 / 2}}{2 \sqrt{\pi}} \mathrm{d} u, \\
\phi_{\mathrm{IG}}^{(n)}(\lambda) & =\frac{(-1)^{n+1}}{2 \sqrt{\pi}} \int_{0}^{\infty} u^{n-3 / 2} \mathrm{e}^{-\lambda u} \mathrm{~d} u \\
& =\frac{(-1)^{n+1}}{2 \sqrt{\pi}} \Gamma\left(n-\frac{1}{2}\right) \lambda^{1 / 2-n} \\
& =\frac{(-1)^{n+1}(2 n-3) !}{2^{2(n-1)}(n-2) !} \lambda^{1 / 2-n} .
\end{aligned}
$$


TABLE 2: PDFs and CDFs of the inverse Gaussian (IG) distribution. Using $k_{i}=10 i$, we give the value of $N$ required to obtain six digits of accuracy $\left(N_{6}\right)$ and 15 digits of accuracy $\left(N_{15}\right)$ at the given values of $x$ using polynomial interpolation. Note that it becomes harder to approximate these functions to a small relative error when they become very small.

\begin{tabular}{ccrccrc}
\hline \multirow{2}{*}{$x$} & \multicolumn{3}{c}{ PDF } & \multicolumn{3}{c}{$\mathrm{CDF}$} \\
\cline { 2 - 7 } & $f_{\mathrm{IG}}(x)$ & $N_{6}$ & $N_{15}$ & $F_{\mathrm{IG}}(x)$ & $N_{6}$ & $N_{15}$ \\
\hline 0.01 & $3.92 \times 10^{-9}$ & 12 & 21 & $1.53 \times 10^{-12}$ & 12 & 21 \\
0.02 & $3.71 \times 10^{-4}$ & 8 & 17 & $5.733 \times 10^{-7}$ & 9 & 17 \\
0.1 & 0.732 & 6 & 14 & 0.025 & 6 & 13 \\
1 & 0.220 & 5 & 11 & 0.480 & 4 & 10 \\
100 & $2.81 \times 10^{-4}$ & 6 & 10 & 0.944 & 4 & 8 \\
1000 & $8.92 \times 10^{-6}$ & 6 & 11 & 0.982 & 3 & 8 \\
\hline
\end{tabular}

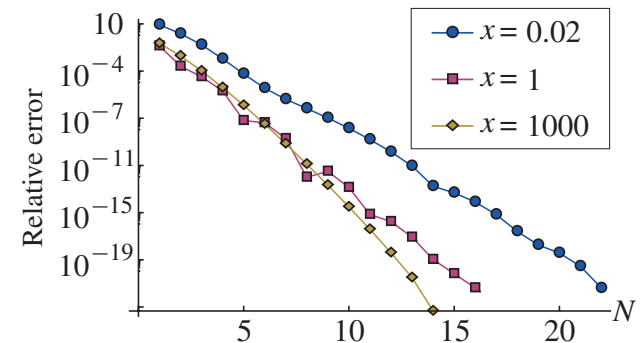

(a)

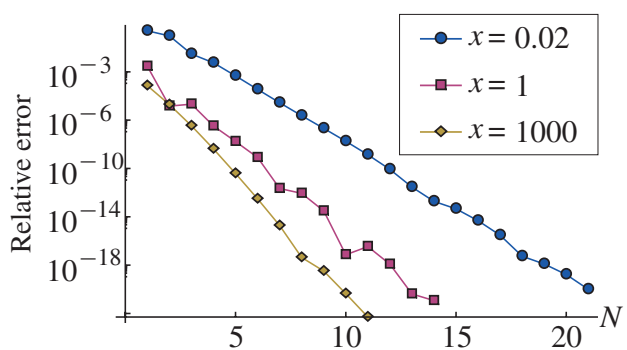

(b)

Figure 3: Plot of the relative error of our method verses the truncation level $N$ for the (a) PDF and (b) $\mathrm{CDF}$ of the inverse Gaussian distribution at three values of $x$.

Table 2 shows the values of $x$ considered, the value of $f_{\mathrm{IG}}(x)$, and the value of $N$ needed to obtain relative errors of $10^{-6}$ and $10^{-15}$. Figure 3 shows how the relative error behaves as a function of $N$ for three values of $x$. Similarly to the $\chi^{2}$ distribution, $N$ is largest when the value of the PDF is very close to 0 .

\section{Applications to infinitely divisible distributions which are not known in closed form}

We will now consider examples of nonnegative infinitely divisible distributions for which the PDF and CDF are not known in closed form. We will compute them numerically using our method. The resulting plots of the PDFs and CDFs are obtained using MATLAB ${ }^{\circledR}$. Each plot was generated in about one second. In order to apply our method, we must first write the Laplace transform in LK form, and then find $\phi(\lambda)$ and the derivatives $\phi^{(n)}(\lambda)$ by computing the integrals in (2.5). In almost every case below, these integrals can be given in 'closed form', which is to say they can at least be written in terms of special functions for which there are efficient methods for computation. We will assume throughout that the drift $a=0$ in (1.1).

The following special functions will appear throughout this section, we provide their definitions here for convenience.

- Gamma function:

$$
\Gamma(a)=\int_{0}^{\infty} z^{a-1} \mathrm{e}^{-z} \mathrm{~d} z, \quad a \neq 0,-1,-2, \ldots
$$


- Lower incomplete gamma function:

$$
\gamma(a, b)=\int_{0}^{b} z^{a-1} \mathrm{e}^{-z} \mathrm{~d} z, \quad b \geq 0, a \neq 0,-1,-2, \ldots
$$

- Upper incomplete gamma function:

$$
\Gamma(a, b)=\int_{b}^{\infty} z^{a-1} \mathrm{e}^{-z} \mathrm{~d} z, \quad b>0, a \in \mathbb{R} .
$$

- Entire exponential integral:

$$
\operatorname{Ein}(a)=\int_{0}^{a} \frac{1-\mathrm{e}^{-z}}{z} \mathrm{~d} z, \quad a \in \mathbb{R}
$$

- Dilogarithm:

$$
L_{2}(a)=\int_{1}^{a} \frac{\log (z)}{z-1} \mathrm{~d} z, \quad a>0 .
$$

See [15, Chapters 25, 37, 43, and 45] for more discussion of these functions and methods for efficient computation. For an integer $n \geq 1$, we have $\Gamma(n)=(n-1)$ ! and

$$
\gamma(n, b)=(n-1) !\left(1-\mathrm{e}^{-b} \sum_{m=0}^{n-1} \frac{b^{m}}{m !}\right)
$$

see [9, Equation 3.351.1].

\subsection{Right-skewed stable distributions}

Here we consider the class of examples with Laplace exponent given by

$$
\phi(\lambda)=\int_{0}^{1} \lambda^{\beta} p(\mathrm{~d} \beta)=\int_{0}^{\infty}\left(1-\mathrm{e}^{-\lambda u}\right)\left(\int_{0}^{1} \frac{u^{-\beta-1}}{\Gamma(-\beta)} p(\mathrm{~d} \beta)\right) \mathrm{d} u,
$$

where $p$ is a measure supported on $(0,1)$. The Lévy measure $\Pi$ has a density given by

$$
\Pi^{\prime}(u)=\int_{0}^{1} \frac{u^{-\beta-1}}{\Gamma(-\beta)} p(\mathrm{~d} \beta) .
$$

Mixtures of this form are considered in [12] and [13], in which these distributions are used in models of anomalous diffusion.

The Laplace exponent is expressed in (4.2) and the derivatives $\phi^{(n)}$ for $n \geq 1$ can be computed using (2.5), a change in the order of integration, and the definition of the gamma function:

$$
\begin{aligned}
\phi^{(n)}(\lambda) & =(-1)^{n+1} \int_{0}^{\infty} u^{n} \mathrm{e}^{-\lambda u}\left(\int_{0}^{1} \frac{u^{-\beta-1}}{\Gamma(-\beta)} p(\mathrm{~d} \beta)\right) \mathrm{d} u \\
& =(-1)^{n+1} \int_{0}^{1} \frac{\lambda^{\beta-n} \Gamma(n-\beta)}{\Gamma(-\beta)} p(\mathrm{~d} \beta), \quad n \geq 1 .
\end{aligned}
$$


Since $n$ takes only integer values, $\Gamma(n-\beta)=\Gamma(-\beta) \prod_{m=0}^{n-1}(m-\beta)$, and so the above can be simplified further as

$$
\begin{aligned}
\phi^{(n)}(\lambda) & =(-1)^{n+1} \int_{0}^{1} \frac{\lambda^{\beta-n} \Gamma(n-\beta)}{\Gamma(-\beta)} p(\mathrm{~d} \beta) \\
& =(-1)^{n+1} \int_{0}^{1} \lambda^{\beta-n} \prod_{m=0}^{n-1}(m-\beta) p(\mathrm{~d} \beta) \\
& =-\frac{1}{\lambda^{n}} \sum_{m=1}^{n} S_{n}^{(m)} \int_{0}^{1} \beta^{m} \lambda^{\beta} p(\mathrm{~d} \beta) \\
& =-\frac{1}{\lambda^{n}} \sum_{m=1}^{n} c_{m}(\lambda) S_{n}^{(m)},
\end{aligned}
$$

where $c_{m}(\lambda)=\int_{0}^{1} \beta^{m} \lambda^{\beta} p(\mathrm{~d} \beta)$ and $\left\{S_{n}^{(m)}\right\}, n \geq 0,0 \leq m \leq n$, are the Stirling numbers of the first kind (see [15, p. 162]). These are such that $\prod_{m=0}^{n-1}(\beta-m)=\sum_{m=1}^{n} S_{n}^{(m)} \beta^{m}$, and can computed with a triangular array similarly to Pascal's triangle using the recursion formula

$$
S_{0}^{(0)}=1, \quad S_{n}^{(0)}=0, \quad n \geq 1, \quad S_{n}^{(m)}=S_{n-1}^{(m-1)}-(n-1) S_{n-1}^{(m)}, \quad n, m \geq 1 .
$$

Let us now consider special cases of $p$ (below, $\delta$ denotes the dirac $\delta$-distribution).

4.1.1. Right-skewed $\alpha$-stable distributions: $p(\mathrm{~d} \beta)=\delta(\beta-\alpha) \mathrm{d} \beta, 0<\alpha<1$. Indeed, an important example of this distribution is the right-skewed $\alpha$-stable distributions, for which $p$ is a point mass at $\beta=\alpha$, with $0<\alpha<1$ (using Proposition 1.2.11 of [20], this distribution corresponds to the stable distribution $\left.S\left(\alpha, 1, \cos (\pi \alpha / 2)^{1 / \alpha}, 0\right)\right)$. These distributions lie in the family of scaling limits for sums of nonnegative i.i.d. random variables with infinite mean. From (4.3), $\phi^{(n)}$ can be computed in various ways:

$$
\phi^{(n)}(\lambda)=(-1)^{n+1} \frac{\lambda^{\alpha-n} \Gamma(n-\alpha)}{\Gamma(-\alpha)}=-\lambda^{\alpha-n} \prod_{m=0}^{n-1}(\alpha-m)=-\lambda^{\alpha-n} \sum_{m=1}^{n} \alpha^{m} S_{n}^{(m)} .
$$

The PDFs and CDFs of the right-skewed $\alpha$-stable distribution are plotted using our method in Figure 4 for several values of $\alpha$. For example, we compute the PDF of a $\frac{1}{2}$-stable distribution at $x=1$ and obtain (in MATHEMATICA) $f(1)=0.219695644733861$. This is exact to 15 decimal places and the computation took approximately half a second. An alternative method for this case is given in [14].

4.1.2. Sums of right-skewed $\alpha$-stable distributions: $p(\mathrm{~d} \beta)=\sum_{j=1}^{r} d_{j} \delta\left(\beta-\alpha_{j}\right) \mathrm{d} \beta, d_{j} \geq 0$, $\sum_{j=1}^{r} d_{j}=1$. The previous case can easily be generalized to weighted sums of independent $\alpha$-stable random variables. From (4.3), it follows that in this case

$$
\begin{aligned}
\phi^{(n)}(\lambda) & =(-1)^{n+1} \sum_{j=1}^{r} d_{j} \frac{\lambda^{\alpha_{j}-n} \Gamma(n-\alpha)}{\Gamma\left(-\alpha_{j}\right)} \\
& =-\sum_{j=1}^{r} d_{j} \lambda^{\alpha_{j}-n} \prod_{m=0}^{n-1}\left(\alpha_{j}-m\right) \\
& =-\sum_{j=1}^{r} d_{j} \lambda^{\alpha_{j}-n}\left(\sum_{m=1}^{n} \alpha_{j}^{m} S_{n}^{(m)}\right) .
\end{aligned}
$$

The PDFs of such distributions are plotted in Figure 5. 

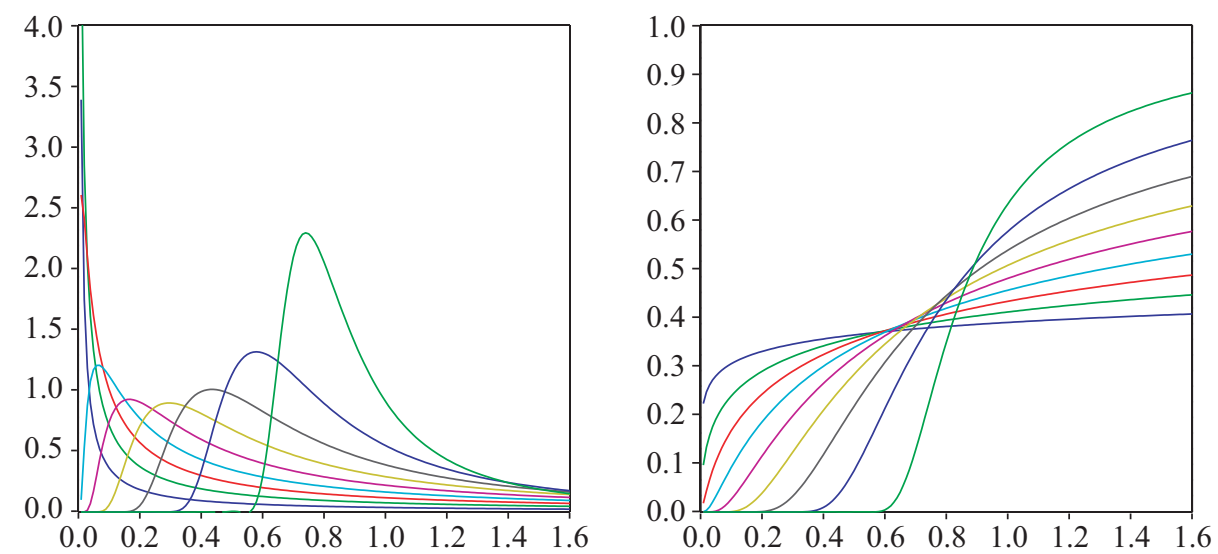

FIGURE 4: Plots of $\alpha$-stable PDFs (left) and CDFs (right) for $\alpha=i / 10, i=1,2, \ldots, 9$. The PDF for $\alpha=0.9$ corresponds to the right-most peak, and $\alpha=0.1$ corresponds to the left-most peak (which is not visible). The CDF with the steepest slope around $x=0.8$ corresponds to $\alpha=0.9$.
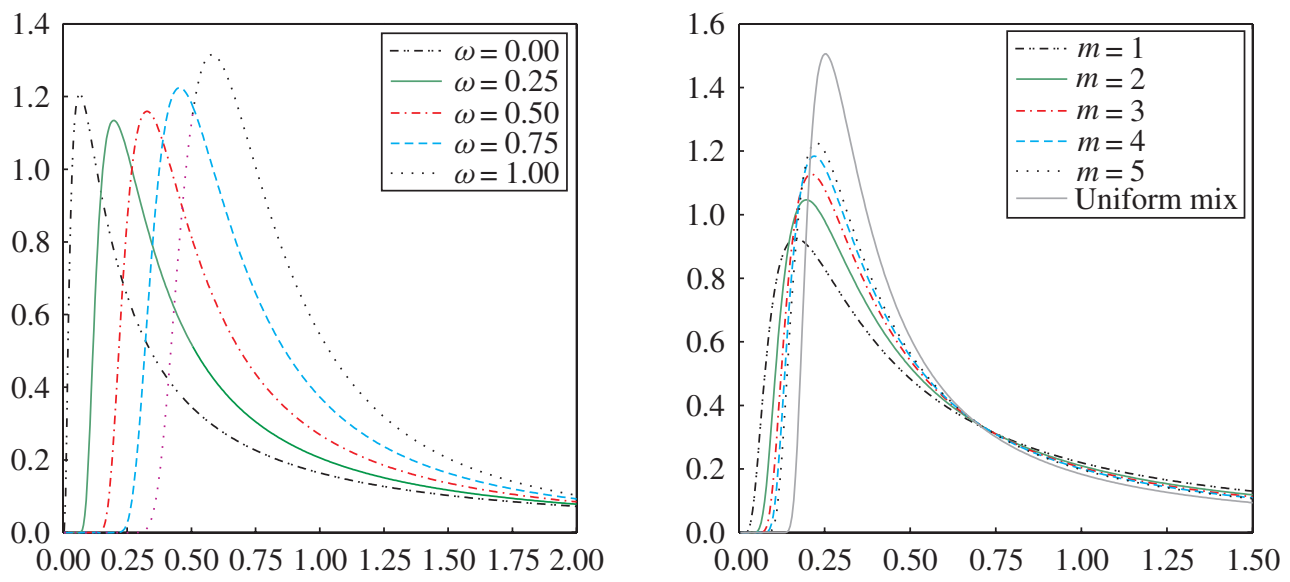

FIGURE 5: (a) A plot of the PDF corresponding to the choice $p_{\omega}(\mathrm{d} u)=(1-\omega) \delta(x-0.4)+\omega \delta(x-0.8)$ for various choices of $0 \leq \omega \leq 1$. By increasing $\omega$, the PDF for $\alpha=0.4$ (on the left) morphs into the PDF for $\alpha=0.8$ (on the right). (b) PDFs corresponding to the sums $p_{m}(\mathrm{~d} u)=(1 / m) \sum_{i=1}^{m} \delta(x-i /(m+1))$ for $m=1,2, \ldots, 5$ together with the uniform continuous mixture on $(0,1)$, which has the highest peak.

4.1.3. A 'uniform mixture' of $\alpha$-stable distributions: $p(\mathrm{~d} \beta)=d \beta, \beta \in(0,1)$. This is an example of a distribution with no finite moments. The Laplace exponent (4.2) is given by

$$
\phi(\lambda)=\int_{0}^{1} \lambda^{\beta} \mathrm{d} \beta=\int_{0}^{1} \mathrm{e}^{\beta \log \lambda} \mathrm{d} \beta=\frac{\lambda-1}{\log \lambda} \quad \text { if } \lambda \neq 1,
$$

and is 1 if $\lambda=1$. Higher derivatives of $\phi$ can be computed using (4.3). The coefficients $c_{m}(\lambda), m=1,2, \ldots, n$, can be computed in a few different ways. First, if $\log \lambda \leq 0$, we have

$$
c_{m}(\lambda)=\int_{0}^{1} \beta^{m} \lambda^{\beta} \mathrm{d} \beta=\int_{0}^{1} \beta^{m} \mathrm{e}^{\beta \log \lambda} \mathrm{d} \beta=\frac{\gamma(1+m,-\log \lambda)}{(-\log \lambda)^{m+1}},
$$


where $\gamma(a, b)$ is the lower incomplete gamma function (see above). Formula (4.4) can still be used for $\log \lambda>0$; however, this requires analytic continuation of $\gamma$ which is not always easy to compute.

As another approach, we compute $c_{m}(\lambda)$ by treating the cases $|\log \lambda|<1$ and $|\log \lambda| \geq 1$ separately. If $|\log \lambda|<1$, note that

$$
c_{m}(\lambda)=\int_{0}^{1} \beta^{m} \mathrm{e}^{\log (\lambda) \beta} \mathrm{d} \beta=\sum_{j=0}^{\infty}(\log \lambda)^{j} \int_{0}^{1} \frac{\beta^{m+j}}{j !} \mathrm{d} \beta=\sum_{j=0}^{\infty} \frac{(\log \lambda)^{j}}{(m+j+1) j !} .
$$

If $|\log \lambda|<1$, taking the first 18 terms in this series gives an absolute error of less than $10^{-15}$. For $|\log \lambda| \geq 1$, the $c_{m}$ s can be computed recursively by applying integration by parts:

$$
\begin{aligned}
c_{0}(\lambda) & =\int_{0}^{1} \mathrm{e}^{\log (\lambda) \beta} \mathrm{d} \beta=\frac{\lambda-1}{\log (\lambda)}, \\
c_{m}(\lambda) & =\int_{0}^{1} \beta^{m} \mathrm{e}^{\log (\lambda) \beta} \mathrm{d} \beta=\frac{\lambda-m c_{m-1}(\lambda)}{\log (\lambda)}, \quad m=1, \ldots, n .
\end{aligned}
$$

Since this procedure involves a division by $\log \lambda$, it should only be used when $|\log \lambda| \geq 1$.

\subsection{Integrals of nonrandom functions with respect to a Poisson random measure}

In this section we consider the large class of nonnegative infinitely divisible distributions which can be expressed in terms of a Poisson stochastic integral of a nonrandom kernel. For a recent review of these integrals, see [16].

Let $(\Omega, \mathcal{F})$ be a probability space, and let $\mathscr{B}$ denote the Borel sigma-field on $\mathbb{R}^{n}$. Let $N(\cdot)$ denote an independently scattered Poisson random measure with control measure $\mu$, that is, $\mu$ is a measure on $\mathscr{B}$ and $N$ is a function $\Omega \times \mathscr{B} \rightarrow \mathbb{Z}^{+} \cup\{0\}$ such that

(i) $N(A) \perp N(B)$ if $A, B \in \mathscr{B}$ are disjoint,

(ii) $N\left(\bigcup_{m=1}^{n} A_{i}\right)=\sum_{m=1}^{n} N\left(A_{i}\right)$ if $A_{i}$ are disjoint,

(iii) for each $A \in \mathscr{B}, N(A)$ has a Poisson distribution with mean $\mu(A)$.

Given such a pair $(N, \mu)$, we can define the stochastic integral

$$
I(g)=\int_{\mathbb{R}^{n}} g(\boldsymbol{x}) N(\mathrm{~d} \boldsymbol{x})
$$

for a function $g$ on $\mathbb{R}^{n}$, for which $\int_{\mathbb{R}^{n}} \min (1, g(\boldsymbol{x})) \mu(\mathrm{d} \boldsymbol{x})<\infty$ and, for our purposes, is nonnegative. In this case, the random variable $I(g)$ is also nonnegative and has Laplace transform

$$
\mathrm{Ee}^{-\lambda I(g)}=\exp \left(-\int_{\mathbb{R}^{n}}\left(1-\mathrm{e}^{-\lambda g(s)}\right) \mu(\mathrm{d} s)\right), \quad \lambda>0 .
$$

In order to compute the PDF and CDF of $I(g)$ using our method, (4.5) must first be rewritten in LK form. In many cases, this can be done with some suitable change of variables $\boldsymbol{u}=\Phi(\boldsymbol{s})$ satisfying $u_{1}=(\Phi(s))_{1}=g(s)$. In this case, (4.5) becomes

$$
\mathrm{Ee}^{-\lambda I(g)}=\exp \left(-\int_{0}^{\infty}\left(1-\mathrm{e}^{-\lambda u_{1}}\right) \Pi_{g}\left(\mathrm{~d} u_{1}\right)\right)
$$


where, for any Borel set $A \subset \mathbb{R}^{+}$, the Lévy measure $\Pi_{g}(A)$ can be expressed formally as

$$
\Pi_{g}(A)=\int_{A \cap \mathbb{R}^{n-1}}\left|J\left(u_{1}, u_{2}, \ldots, u_{n}\right)\right|\left(\mu \circ \Phi^{-1}\right)\left(\mathrm{d} u_{1}, \mathrm{~d} u_{2}, \ldots, \mathrm{d} u_{n}\right),
$$

where $|J|$ is the Jacobian $\partial \Phi^{-1}\left(u_{1}, \ldots, u_{n}\right) / \partial\left(u_{1}, \ldots, u_{n}\right)$.

To illustrate this, let us now focus on special cases, in dimensions $n=1$ and $n=2$, where this change of variable can be made and our method applied.

4.2.1. One-dimensional Poisson integral. Assume that $n=1$ and that the integrand $g$ is a monotone, nonnegative function with inverse $g^{-1}$. In this case, (4.5) can be rewritten in LK form using the change of variables $u=g(s)$ :

$$
\mathrm{Ee}^{-\lambda I(g)}=\exp \left(-\int_{0}^{\infty}\left(1-\mathrm{e}^{-\lambda u}\right) \Pi_{g}(\mathrm{~d} u)\right) .
$$

Since $u=g^{-1}(g(u))$, we get $1=\left(g^{\prime} \circ g^{-1}\right)(u)\left(g^{-1}\right)^{\prime}(u)$, and, hence,

$$
\Pi_{g}(\mathrm{~d} u)=\frac{\mathbf{1}_{g((0, \infty))}(u)}{\left|\left(g^{\prime} \circ g^{-1}\right)(u)\right|}\left(\mu \circ g^{-1}\right)(\mathrm{d} u)
$$

where $g((0, \infty))$ denotes the image of $(0, \infty)$ under $g$. Suppose that we want to get the PDF and $\mathrm{CDF}$ of

$$
I(g)=\int_{0}^{\infty} \mathrm{e}^{-s / \eta} N(\mathrm{~d} s),
$$

where $g(s)=\mathrm{e}^{-s / \eta}, \eta>0$, is a parameter and the control measure $\mu$ is Lebesgue. In this case, a simple calculation shows that (4.6) becomes

$$
\Pi_{g}(\mathrm{~d} u)=\left(\frac{\eta}{u}\right) \mathbf{1}_{(0,1]}(u) \mathrm{d} u
$$

Thus, for this example,

$$
\phi(\lambda)=\int_{0}^{1}\left(1-\mathrm{e}^{-\lambda u}\right) \frac{\eta}{u} \mathrm{~d} u=\eta \operatorname{Ein}(\lambda)
$$

where $\operatorname{Ein}(\lambda)$ is the entire exponential integral defined earlier. The derivatives $\phi^{(n)}(\lambda)$ for $n \geq 1$ can be given in closed form, i.e.

$$
\begin{aligned}
\phi^{(n)}(\lambda) & =(-1)^{n+1} \eta \int_{0}^{1} u^{n-1} \mathrm{e}^{-\lambda u} \mathrm{~d} u \\
& =\frac{(-1)^{n+1} \eta}{\lambda^{n}} \gamma(n, \lambda) \\
& =\frac{(-1)^{n+1} \eta}{\lambda^{n}}\left((n-1) !-\mathrm{e}^{-\lambda} \sum_{m=0}^{n-1} \frac{(n-1) !}{m !} \lambda^{m}\right),
\end{aligned}
$$

where the last equality follows from (4.1). We have plotted the PDF and CDF of the random variable $I\left(\mathrm{e}^{-s / \eta}\right)$ for various values of $\eta$ in Figure 6 using our method. Note that, since the range of integration here is $(0, \infty)$, the method described in [23] does not readily apply. 

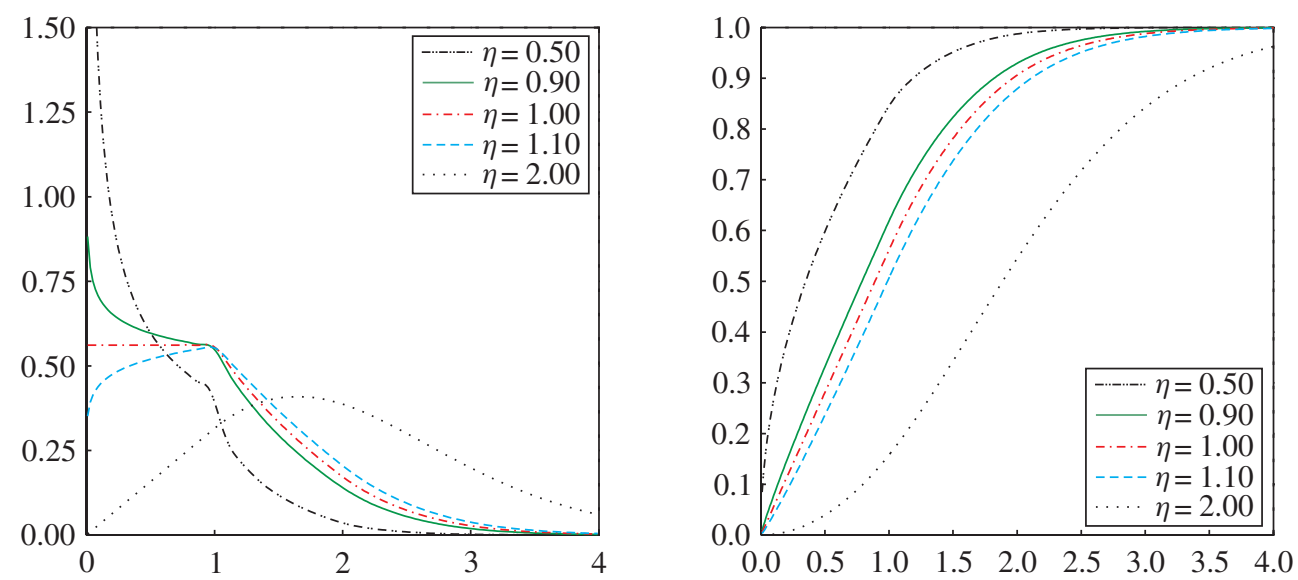

FIGURE 6: Plots of the PDF (left) and CDF (right) for the Poisson stochastic integral $I\left(\mathrm{e}^{-s / \eta}\right)$ with various values of the parameter $\eta$, which plays the role of a shape parameter. The PDF for $\eta=0.5$ is the highest on the left.

4.2.2. Integration with respect to a nonnegative Lévy process with a nonnegative kernel. In this example we generalize the previous case by looking at integration with respect to a nonnegative Lévy process, or, equivalently, a one-dimensional nonnegative infinitely divisible random measure $L$ with control measure $\mu$. Here $L$ is a random measure which satisfies the same conditions as the Poisson random measure $N$, except condition (iii) is replaced by the following.

(iii) $^{\prime}$ There exists a Lévy measure $\Pi$ such that, for any $A \in \mathcal{B}$, the distribution of $L(A)$ has Laplace transform

$$
\mathrm{Ee}^{-\lambda L(A)}=\exp \left(-\mu(A) \int_{0}^{\infty}\left(1-\mathrm{e}^{-\lambda u}\right) \Pi(\mathrm{d} u)\right) .
$$

Note that the Poisson random measure $N$ corresponds to the choice $\Pi(\mathrm{d} u)=\delta(u-1) \mathrm{d} u$. For a given function $g$, the stochastic integral $I_{L}(g)$ can be defined in terms of a two-dimensional Poisson stochastic integral:

$$
I_{L}(g)=\int_{0}^{\infty} g(s) L(\mathrm{~d} s) \equiv \int_{0}^{\infty} \int_{0}^{\infty} u g(z) N(\mathrm{~d} u, \mathrm{~d} z) .
$$

Here the control measure of $N$ is given now by $\Pi(\mathrm{d} u) \mu(\mathrm{d} z)$. Observe that the kernel $g$ must now satisfy $\int_{0}^{\infty} \int_{0}^{\infty} \min (1, u g(z)) \Pi(\mathrm{d} u) \mu(\mathrm{d} z)<\infty$.

Assume now for simplicity that $\Pi(\mathrm{d} u)=\Pi(u) \mathrm{d} u$ for some function $\Pi$ and that $g$ is a nonnegative monotone function with inverse $g^{-1}$. In this case, we can obtain the LK form corresponding to $I_{L}$ :

$$
\begin{aligned}
\mathrm{Ee}^{-\lambda I_{L}(g)} & =\exp \left(-\int_{0}^{\infty} \int_{0}^{\infty}\left(1-\mathrm{e}^{-\lambda u g(z)}\right) \Pi^{\prime}(u) \mathrm{d} u \mu(\mathrm{d} z)\right) \\
& =\exp \left(-\int_{0}^{\infty}\left(1-\mathrm{e}^{-\lambda v}\right) \Pi_{g}^{\prime}(v) \mathrm{d} v\right) .
\end{aligned}
$$


Here we have made the change of variables $v=u g(z), z^{\prime}=z$, and the measure $\Pi_{g}(\mathrm{~d} v)$ is given by

$$
\Pi_{g}^{\prime}(v)=\int_{0}^{\infty} \Pi^{\prime}\left(\frac{v}{g\left(z^{\prime}\right)}\right) \frac{1}{g\left(z^{\prime}\right)} \mu\left(\mathrm{d} z^{\prime}\right) .
$$

To demonstrate our method in this case, consider

$$
I_{L}(g)=\int_{0}^{\infty} \mathrm{e}^{-z / \eta} L(\mathrm{~d} z)=\int_{0}^{\infty} \int_{0}^{\infty} u \mathrm{e}^{-z / \eta} N(\mathrm{~d} u, \mathrm{~d} z),
$$

where $g(z)=\mathrm{e}^{-z / \eta}$ with $\eta>0, \mu$ is Lebesgue, and $\Pi(\mathrm{d} u)=\kappa u^{-1} \mathrm{e}^{-u / \theta} \mathrm{d} u$, which is the Lévy measure corresponding to the gamma distribution with shape $\kappa>0$ and scale $\theta>0$ (see [3, Example 1.3.22]). With the change of variable $w=u \mathrm{e}^{z / \eta},(4.8)$ implies that

$$
\Pi_{g}^{\prime}(u)=\int_{0}^{\infty} \Pi^{\prime}\left(u \mathrm{e}^{z / \eta}\right) \mathrm{e}^{z / \eta} \mathrm{d} z=\frac{\eta \kappa}{u} \int_{u}^{\infty} w^{-1} \mathrm{e}^{-w / \theta} \mathrm{d} w=\frac{\eta \kappa}{u} \Gamma\left(0, \frac{u}{\theta}\right) .
$$

We can now compute the corresponding Laplace exponent for this case:

$$
\begin{aligned}
\phi(\lambda) & =\int_{0}^{\infty}\left(1-\mathrm{e}^{-\lambda u}\right) \Pi_{g}^{\prime}(u) \mathrm{d} u \\
& =\eta \kappa \int_{0}^{\infty} \frac{1-\mathrm{e}^{\lambda u}}{u} \Gamma\left(0, \frac{u}{\theta}\right) \mathrm{d} u \\
& =\eta \kappa \int_{0}^{\infty} \int_{0}^{\lambda} \mathrm{e}^{-u t} \int_{u}^{\infty}\left(w^{-1} \mathrm{e}^{-w / \theta}\right) \mathrm{d} w \mathrm{~d} t \mathrm{~d} u \\
& =\eta \kappa \int_{0}^{\lambda}\left(\int_{0}^{\infty} \int_{0}^{w}\left(w^{-1} \mathrm{e}^{-u t-w / \theta}\right) \mathrm{d} u \mathrm{~d} w\right) \mathrm{d} t \\
& =\eta \kappa \int_{0}^{\lambda} \frac{1}{t}\left(\int_{0}^{\infty} w^{-1} \mathrm{e}^{-w / \theta}\left(1-\mathrm{e}^{-w t}\right) \mathrm{d} w\right) \mathrm{d} t \\
& =\eta \kappa \int_{0}^{\lambda} \frac{1}{t} \int_{0}^{\infty}\left(\mathrm{e}^{-w / \theta} \int_{0}^{t} \mathrm{e}^{-w s} \mathrm{~d} s\right) \mathrm{d} w \mathrm{~d} t \\
& =\eta \kappa \int_{0}^{\lambda} \frac{1}{t}\left(\int_{0}^{t} \int_{0}^{\infty} \mathrm{e}^{-w(s+1 / \theta)} \mathrm{d} w \mathrm{~d} s\right) \mathrm{d} t \\
& =\eta \kappa \int_{0}^{\lambda} \frac{\log (1+t \theta)}{t} \mathrm{~d} t \\
& =\eta \kappa L_{2}(1+\lambda \theta) .
\end{aligned}
$$

The derivatives of $\phi$ can also be computed exactly in this case. Using (2.5) and (4.1),

$$
\begin{aligned}
\phi^{(n)}(\lambda) & =(-1)^{n+1} \eta \kappa \int_{0}^{\infty} u^{n-1} \mathrm{e}^{-\lambda u} \Gamma\left(0, \frac{u}{\theta}\right) \mathrm{d} u \\
& =(-1)^{n+1} \eta \kappa \int_{0}^{\infty} u^{n-1} \mathrm{e}^{-\lambda u} \int_{u}^{\infty}\left(w^{-1} \mathrm{e}^{-w / \theta}\right) \mathrm{d} w \mathrm{~d} u \\
& =(-1)^{n+1} \eta \kappa \int_{0}^{\infty} w^{-1} \mathrm{e}^{-w / \theta}\left(\int_{0}^{w} u^{n-1} \mathrm{e}^{-\lambda u} \mathrm{~d} u\right) \mathrm{d} w \\
& =(-1)^{n+1} \eta \kappa \int_{0}^{\infty} w^{-1} \mathrm{e}^{-w / \theta}\left(\frac{(n-1) !}{\lambda^{n}}-\mathrm{e}^{-w \lambda} \sum_{m=0}^{n-1} \frac{(n-1) !}{m !} \frac{w^{m}}{\lambda^{n-m}}\right) \mathrm{d} w
\end{aligned}
$$



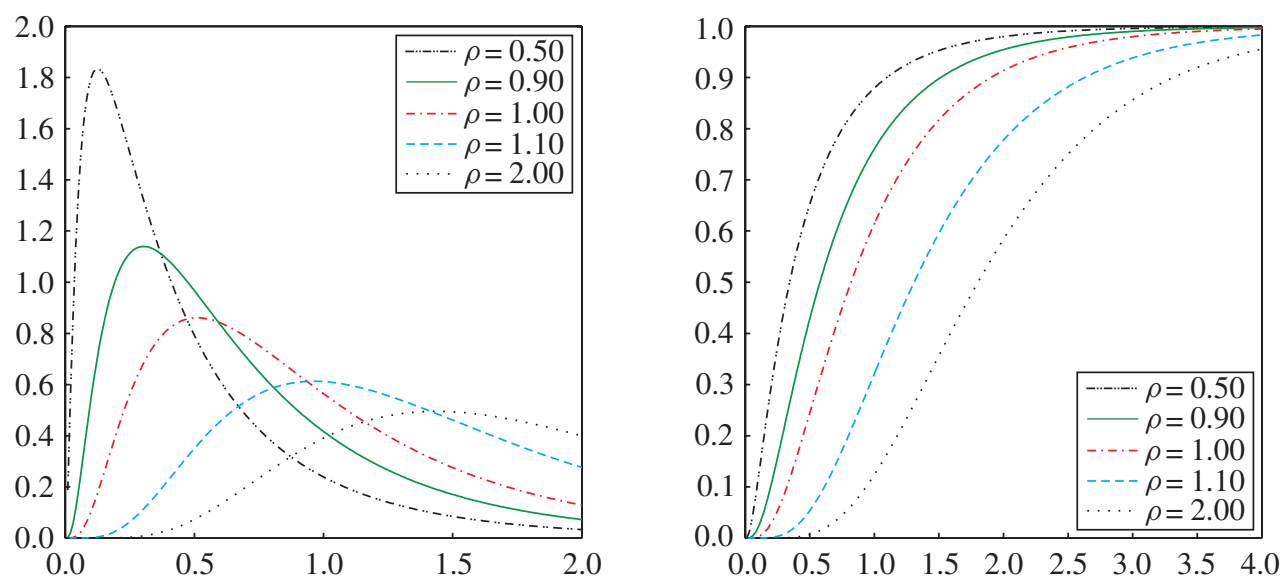

Figure 7: Plots of the PDF (left) and CDF (right) for the Poisson stochastic integral $I_{L}\left(\mathrm{e}^{-s / \eta}\right)$ with various values of the parameter $\eta$.

$$
\begin{aligned}
= & (-1)^{n+1} \eta \kappa \frac{(n-1) !}{\lambda^{n}} \\
& \times\left(\int_{0}^{\infty} w^{-1} \mathrm{e}^{-w / \theta}\left(1-\mathrm{e}^{-w / \theta}\right) \mathrm{d} w-\sum_{m=1}^{n-1} \frac{\lambda^{m}}{m !} \int_{0}^{\infty} w^{m-1} \mathrm{e}^{-w(\lambda+1 / \theta)} \mathrm{d} w\right) \\
= & (-1)^{n+1} \eta \kappa \frac{(n-1) !}{\lambda^{n}}\left(\log (1+\lambda \theta)-\sum_{m=1}^{n-1} \frac{(\lambda \theta)^{m}}{m(1+\lambda \theta)^{m}}\right),
\end{aligned}
$$

where the first integral in the second to last line above is computed as in (4.9). Alternatively, $\phi^{(n)}(\lambda)$ above can also be given simply in terms of the Gauss hypergeometric function ${ }_{2} F_{1}(\alpha, \beta, \gamma, x)$ (see [9, Equation 6.455.1, p. 657]):

$$
\phi^{(n)}(\lambda)=(-1)^{n+1} \eta \kappa \frac{\theta^{n}(n-1) !}{n}{ }_{2} F_{1}(n, n, n+1, \lambda \theta) .
$$

In Figure 7 we have plotted the PDF and CDF of (4.7) for $\theta=1$ and various values of the product $\rho=\eta \kappa$ using our method.

\section{Appendix A. Implementation}

We provide here an outline of the algorithm for computing the PDF and CDF based upon using the recursive formula (2.3) and the polynomial extrapolation method. This method is implemented in MATLAB and MATHEMATICA and the code is available on request from the authors. The method using rational extrapolation is also an option in the MATLAB implementation, but is not described here.

Algorithm A.1. (Algorithm for computing the PDF $f$ at $x>0$.)

1. Choose the sequence $1=k_{0}<k_{1}<k_{2}<\cdots<k_{N}$ and a relative error tolerance $\varepsilon>0$. We found $k_{j}=10 j$, with $N$ lying around 8 to be effective for $\varepsilon=10^{-6}$. Initialize the 
following arrays.

\begin{tabular}{ccc}
\hline Variable & Size & Purpose \\
\hline$D_{\phi}$ & $\left(k_{N}-1\right) \times N$ & Holds derivatives of $\phi$ \\
$D_{\psi}$ & $k_{N} \times N$ & Holds derivatives of $\psi$ \\
$P$ & $1 \times N$ & Holds Post-Widder approximations of $f$ \\
$f$ & $1 \times N$ & Holds extrapolated approximations of $f$ \\
\hline
\end{tabular}

Set $j=1$.

2. Compute $\left(D_{\psi}\right)_{1, j}$ as

$$
\left(D_{\psi}\right)_{1, j}=\psi\left(\frac{k_{j}}{x}\right) \text {. }
$$

3. For $i=k_{j-1}, \ldots, k_{j}-1$, compute the $i$ th row of $D_{\phi}$ as

$$
\left(D_{\phi}\right)_{i, j}=\left\{\begin{array}{ll}
\phi^{(i)}\left(k_{j} / x\right), & i \leq k_{j}, \\
0, & \text { otherwise, }
\end{array} \quad j=1,2, \ldots, N .\right.
$$

We compute $\phi^{(i)}$ using the integrals in (2.5). The $(i+1)$ th row of $D_{\psi}$ is then computed as

$$
\left(D_{\psi}\right)_{i+1, j}= \begin{cases}\sum_{r=1}^{i}\left(\begin{array}{l}
i \\
r
\end{array}\right)\left(D_{\psi}\right)_{r, j}\left(D_{\phi}\right)_{i+1-r, j}, & i \leq k_{j}, \quad j=1,2, \ldots, N . \\
0, & \text { otherwise, }\end{cases}
$$

4. Compute the $j$ th Post-Widder approximation as

$$
P_{j}=\frac{(-1)^{k_{j}-1}}{\left(k_{j}-1\right) !}\left(\frac{k_{j}}{x}\right)^{k_{j}}\left(D_{\psi}\right)_{k_{j}, j} .
$$

5. If $j=1$, set $f_{1}=P_{1}, j=2$, and go to step 3. Otherwise, compute the $j$ th extrapolation as

$$
f_{j}=\sum_{r=1}^{j} c_{r} P_{r}, \quad \text { where } \quad c_{r}=\prod_{\ell=1, \ell \neq r}^{r} \frac{k_{r}}{k_{\ell}-k_{r}} .
$$

Set $\alpha=1+2\left(k_{j} / k_{1}+1\right)^{-1}$. If $\left|(1+\alpha)\left(f_{j}-f_{j-1}\right)\right| / f_{j}<\varepsilon$ or if $j=N$, return $f_{j}$. Otherwise, set $j=j+1$ and go to step 3 .

The method for computing the CDF is similar, except that (2.6) is used instead for step 4.

Algorithm A.2. (Algorithm for computing the CDF $F$ at $x>0$.) Follow the steps for the PDF computation above, except replace step 4 with

$4^{\star}$. Compute the $j$ th Post-Widder approximation as

$$
P_{j}=\sum_{r=0}^{k_{j}-1} \frac{(-1)^{k_{j}+r-1}}{\left(k_{j}-r-1\right) !}\left(\frac{k_{j}}{x}\right)^{k_{j}-r-1}\left(D_{\psi}\right)_{k_{j}-r, j} .
$$

We will conclude with a collection of remarks regarding the implementation of this procedure. 
Remarks. 1. Binomial coefficients are reused multiple times in step 3. We found it useful to compute and store rows of Pascal's triangle as needed.

2. In MATLAB, all computations above can be 'vectorized' to maximize speed.

3. It is essential to compute the derivatives of $\phi$ to as many significant digits of accuracy in step 3 as you want in your final result. In many examples, a closed form for $\phi^{(n)}$ can be found.

4. To avoid overflow, ratios of large numbers like those seen in steps 4 and $4^{\star}$ should be computed to incorporate reduction. For example, using identities such as

$$
\frac{1}{\left(k_{j}-1\right) !}\left(\frac{k_{j}}{x}\right)^{k_{j}}=\exp \left(k_{j} \log \left(\frac{k_{j}}{x}\right)-\sum_{j=1}^{k_{j}-1} \log (j)\right)
$$

will alleviate overflow.

5. In double precision arithmetic, it is often impossible to take $k_{N}$ much bigger than 100 as underflow, overflow, and numerical instability become unavoidable. In most cases, adequate convergence is met much before such large $k$ values are needed; however, if one must go further, it will become necessary to work with a high-precision arithmetic package. MATHEMATICA, for example, has powerful multiprecision capabilities. If one requires more speed, a well developed and documented library for multiple precision arithmetic for $\mathrm{C}++/$ Fortran is available at http://crd.lbl.gov/ dhbailey/mpdist.

6. When the true value of $f$ is very close to $0\left(f(x) \ll 10^{-8}\right)$, the extrapolation procedure might 'overshoot' 0 and return a negative value for the density. In this case, 0 is a good approximation as you can be sure that the PDF or CDF takes an extremely small value in this case, and similarly when the CDF takes a value greater than 1 .

\section{References}

[1] Aваte, J. And Whitt, W. (1992). The Fourier-series method for inverting transforms of probability distributions. Queueing Systems 10, 5-87.

[2] Aвate, J., Choudhury, G. L. And Whitt, W. (2000). An introduction to numerical transform inversion and its application to probability models. In Computational Probability, ed. W. K. Grassman, Kluwer, Norwell, NA, pp. 257-323.

[3] Applebaum, D. (2004). Lévy Processes and Stochastic Calculus. Cambridge University Press.

[4] Bertoin, J. (1996). Lévy Processes. Cambridge University Press.

[5] Bertoin, J. (1999). Subordinators: examples and applications. In Lectures on Probability Theory and Statistics (Saint-Flour, 1997; Lecture Notes Math. 1717), Springer, Berlin, pp.1-91.

[6] Bulirsch, R. AND Stoer, J. (1966). Asymptotic upper and lower bounds for results of extrapolation methods. Numer. Math, 8, 93-104.

[7] Feller, W. (1971). An Introduction to Probability Theory and Its Applications, Vol. 2, 2nd edn. John Wiley, New York.

[8] Frolov, G. A. and Kitaev, M. Y. (1998). Improvement of accuracy in numerical methods for inverting Laplace transforms based on the Post-Widder formula. Comput. Math. Appl. 36, 23-34.

[9] Gradshteyn, I. S. And Ryzhik, I. M. (2007). Table of Integrals, Series, and Products, 7th edn. Elsevier/ Academic Press, Amsterdam.

[10] Jagerman, D. L. (1982). An inversion technique for the Laplace transform. Bell System Tech. J. 61, 1995-2002.

[11] JoyCe, D. C. (1971). Survey of extrapolation processes in numerical analysis. SIAM Rev. 13, 435-490.

[12] Kovacs, M. and Meerschaert, M. M. (2006). Ultrafast subordinators and their hitting times. Publ. L'Institut Math. 94, 193-206.

[13] Meerschaert, M. M. and Scheffler, H.-P. (2006). Stochastic model for ultraslow diffusion. Stoch. Process. Appl. 116, 1213-1235. 
[14] Nolan, J. P. (1997). Numerical calculation of stable densities and distribution functions. Commun. Statist. Stoch. Models 13, 759-774.

[15] Oldham, K., Myland, J. And Spanier, J. (2009). An Atlas of Functions, 2nd edn. Springer, New York.

[16] Peccati, G. and Taqqu, M. S. (2011). Wiener Chaos: Moments, Cumulants and Diagrams. Springer.

[17] Pitman, J. (2006). Combinatorial Stochastic Processes (Lecture Notes Math 1875). Springer, Berlin.

[18] Riordan, J. (2002). An Introduction to Combinatorial Analysis. Dover Publications, Mineola, NY.

[19] Roman, S. (1984). The Umbral Calculus (Pure Appl. Math. 111). Academic Press, New York.

[20] Samorodnitsky, G. and TaquU, M. S. (1994). Stable Non-Gaussian Random Processes. Chapman \& Hall, New York.

[21] Sato, K. I. (1999). Lévy Processes and Infinitely Divisible Distributions (Camb. Stud. Adv. Math. 68). Cambridge University Press.

[22] Steutel, F. W. and van Harn, K. (2004). Infinite Divisibility of Probability Distributions on the Real Line (Monogr. Textbooks Pure Appl. Math. 259). Marcel Dekker, New York.

[23] Veillette, M. and Taqqu, M. S. (2010). Distribution functions of Poisson random integrals: Analysis and computation. Preprint. Available at http://arxiv.org/abs/1004.5338v1.

[24] Veillette, M. and Taqqu, M. S. (2010). Numerical computation of first-passage times of increasing Lévy processes. Methodology Comput. Appl. Prob. 12, 695-729. 